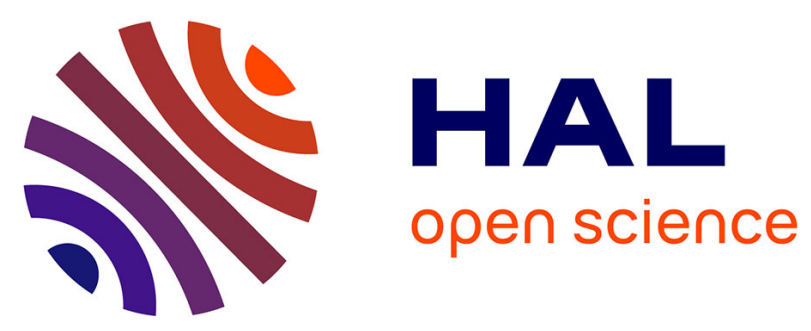

\title{
Effects of Pressure Oscillations on Drainage in an Elastic Porous Medium
}

\author{
M. Jankov, G. Løvoll, H. A Knudsen, Knut Jørgen J Måløy, R. Planet, \\ Renaud Toussaint, Eirik Grude Flekkøy
}

\section{- To cite this version:}

M. Jankov, G. Løvoll, H. A Knudsen, Knut Jørgen J Måløy, R. Planet, et al.. Effects of Pressure Oscillations on Drainage in an Elastic Porous Medium. Transport in Porous Media, 2010, 84 (3), pp.569-585. 10.1007/s11242-009-9521-z . insu-02377796

\section{HAL Id: insu-02377796 https://hal-insu.archives-ouvertes.fr/insu-02377796}

Submitted on 24 Nov 2019

HAL is a multi-disciplinary open access archive for the deposit and dissemination of scientific research documents, whether they are published or not. The documents may come from teaching and research institutions in France or abroad, or from public or private research centers.
L'archive ouverte pluridisciplinaire HAL, est destinée au dépôt et à la diffusion de documents scientifiques de niveau recherche, publiés ou non, émanant des établissements d'enseignement et de recherche français ou étrangers, des laboratoires publics ou privés. 


\title{
Effects of pressure oscillations on drainage in an elastic porous medium
}

\author{
M. Jankov ${ }^{1}$ (mihailo.jankov@fys.uio.no), G. Løvoll ${ }^{1}, \mathrm{H}$. A. \\ Knudsen ${ }^{1}$, K. J. Måløy ${ }^{1}$, R. Planet ${ }^{2}$, R. Toussaint ${ }^{3}$ and E. G. \\ Flekkøy ${ }^{1}$ \\ ${ }^{1}$ Department of Physics, University of Oslo, P.O. Box 1048 Blindern, NO-0316, \\ Oslo, Norway \\ ${ }^{2}$ Departament d'Estructura $i$ Constituents de la Matèria, Universitat de Barcelona, \\ Marti i Franquès 1, E-08028, Barcelona, Spain \\ ${ }^{3}$ Institut de Physique du Globe de Strasbourg, CNRS, Université Louis Pasteur, 5 \\ rue Descartes, 67084 Strasbourg Cedex, France
}

\begin{abstract}
The effects of seismic stimulation on the flow of two immiscible fluids in an elastic synthetic porous medium is experimentally investigated. A wetting fluid is slowly evacuated from the medium, while a pressure oscillation is applied on the injected non-wetting fluid. The amplitude and frequency of the pressure oscillations as well as the evacuation speed are kept constant throughout an experiment. The resulting morphology of the invading structure is found to be strongly dependent on the interplay between the amplitude and the frequency of the applied pressure oscillations and the elasticity of the porous medium. Different combinations of these properties yield morphologically similar structures, allowing a classification of structures that is found to depend on a proposed dimensionless number.
\end{abstract}

Keywords: drainage displacement, elasticity, porous material, stimulated flow, twophase flow, capillary forces, viscous forces

\section{Introduction}

The observed increase of oil recovery in the production wells affected by seismic activity triggered the research of alternative ways in stimulating removal of the residual oil in reservoirs (Beresnev and Johnson (1994), Beresnev et al. (2005)). The attempts that have been made so far in the investigation on a possible increase in the extraction of organic hydro-carbons range from the use of surfactants in order to reduce the surface tension between the phases (Fountain et al. (1991), Pennell et al. (1994)) to the use of elastic waves (Beresnev and Johnson (1994), Reddi and Challa (1994), Roberts et al. (2003)) and ultra-sonic waves (Roberts et al. (2001)). The research has been developing in both experimental (Roberts et al. (2001), Li et al. (2005)) and theoretical 
directions (Hilpert et al. (2000), Iassonov and Beresnev (2003), Hilpert (2007), Pride et al. (2008)).

The objective of this study is an experimental investigation of the impacts of seismic stimulation on horizontal flow of two immiscible fluids in a small scale elastic porous medium. The properties of this stimulated drainage process are compared with established knowledge and placed in the context of non-stimulated drainage. Recall that, generally, a non-stimulated displacement in the horizontal plane is controlled by capillary and viscous forces (Lenormand et al. (1983)). As one of the phases is being removed from the medium, the other one advances creating the invasion pattern. Depending on which force dominates the flow and the properties of the fluids (e.g. viscosity and density contrast, wettability) different geometries of the invading structure can be achieved (Lenormand et al. (1988), Toussaint et al. (2005), Løvoll et al. (2005)).

A horizontal displacement of a wetting by a nonwetting fluid in the capillary regime results in capillary fingering structures that are well reproduced by the invasion percolation (IP) algorithm (Chandler et al. (1982), Wilkinson and Willemsen (1983), Lenormand and Zarcone (1985), Lenormand et al. (1988), Lenormand and Zarcone (1989)). Fast displacement of the same two fluids is governed by the viscous forces. An invasion of a fluid that is more viscous than the defending fluid, results in a stable displacement (Dullien (1992), Lenormand et al. (1988), Aker et al. (2000)). Injecting a less viscous non-wetting fluid gives viscous fingering (Saffman and Taylor (1958), Måløy et al. (1985), Lenormand et al. (1988), Løvoll et al. (2004)). However, at intermediate outflow velocities, the invading patterns exhibit similarity with the IP patterns on a small scale, whereas on larger scales the displacement structures resemble geometries characteristic for the viscous fingering regime (Toussaint et al. (2005)). In a non-horizontal system, gravity and density contrast between the fluids result in a variety of displacement structures (Wilkinson (1984), Birovljev et al. (1991), Wagner et al. (1997), Méheust et al. (2002)).

In this study the focus is shifted from a non-stimulated flow to stimulated drainage in a horizontally placed elastic porous medium. The stimulation consists of a pressure oscillation applied to the non-wetting fluid while the wetting fluid is being slowly removed from the porous matrix. Systematically changing the amplitude and the frequency of the pressure oscillations, different morphologies of the invading cluster can be achieved. The shape changes from invasion-percolation like, through different finger-like structures ending with a fragmented and cloud-like structure as the amplitude of the oscillations are increased. 
The observed change in the flow patterns is strongly coupled to the elasticity of the system as it responds to the pressure perturbations.

To put the oscillatory pressure stimulation in a field context we look into seismic waves. A seismic wave consists of two components, the so-called S- and P-wave. The S-wave corresponds to a sinusoidal translation wave of the rock matrix. In a system with fluids and matrix of different densities the S-wave generates body forces (Pride et al. (2008)). We focus on the compressional component of the seismic wave, the $\mathrm{P}$-wave, and its effects on the soft inclusions in the system. A passing $\mathrm{P}$-wave corresponds to a homogeneous compression or dilatation, over scales smaller than the wave length.

When a large scale homogeneous compression is imposed from the boundary over a system with soft inclusions, the stress variation will be larger around this inclusions (Eshelby (1957)). If a soft fluid, like air, is trapped in a rock, these pockets will amplify the amplitude of the pressure oscillation corresponding to the $\mathrm{P}$-wave. This pressure oscillation larger than in the surrounding, resonating due to the compressibility heterogeneity, will emit secondary pressure waves penetrating in the surrounding medium like a diffusion wave, according to Biot's theory (Biot 1962). This is the situation analogous to our experiments.

The manuscript is organized as follows. Section 2 presents the experimental setup and provides the experimentally measured elasticity properties of the porous medium. The boundary conditions are presented in detail as they are novel to this setup as compared to work on non-stimulated systems. Section 3 is a theoretical treatment of the physics of the process. The elastic properties of the model are discussed, and a dimensionless number that serves as relevant parameter in the system is given. Section 4 gives in-depth experimental results and, based on visual appearance, proposes a classification of the obtained structures.

\section{Experimental setup and methodology}

The core of the experimental model is the porous matrix and the tubing system, through which the flow is controlled. A schematic view of the model is shown in Figure 1.

\subsection{Porous MATRIX - Elastic Properties}

The experiments are performed with a quasi-two-dimensional laboratory made porous medium. It consists of a randomly distributed monolayer of $1 \mathrm{~mm}$ diameter glass beads between two sheets of transparent 
contact paper. The size of the contact paper sheets is $50 \mathrm{~cm} \times 50 \mathrm{~cm}$. The beads are poured on the lower contact paper sheet to cover the entire area of it. The active area of the porous matrix, a $35 \mathrm{~cm} \times 20 \mathrm{~cm}$ rectangle, is sealed off by silicon glue before adhering the top contact paper sheet onto the beads. The top panel of the porous matrix is then slit to provide the access and the escape openings for fluids. A dissected view of the porous matrix is given in Figure 2. The porosity and the permeability of the porous medium are measured to be $\phi_{0}=0.63$ and $\kappa=1.9 \times 10^{-5} \mathrm{~cm}^{2}$, respectively, and the volume of a single pore is approximately $1 \mathrm{~mm}^{3}$. The upper contact paper is glued to a transparent $50 \times 50 \times 2 \mathrm{~cm}^{3}$ Plexiglas plate. There are two channels, $0.5 \mathrm{~cm}$ wide and $0.8 \mathrm{~cm}$ deep on the bottom of the Plexiglas plate that are aligned with the openings on the matrix, allowing access to the model volume. The Plexiglas plate with the porous layer is clamped to a 'water cushion', the pressure from which impedes horizontal movement of the glass beads and helps in keeping the temperature of the system homogeneous, thus

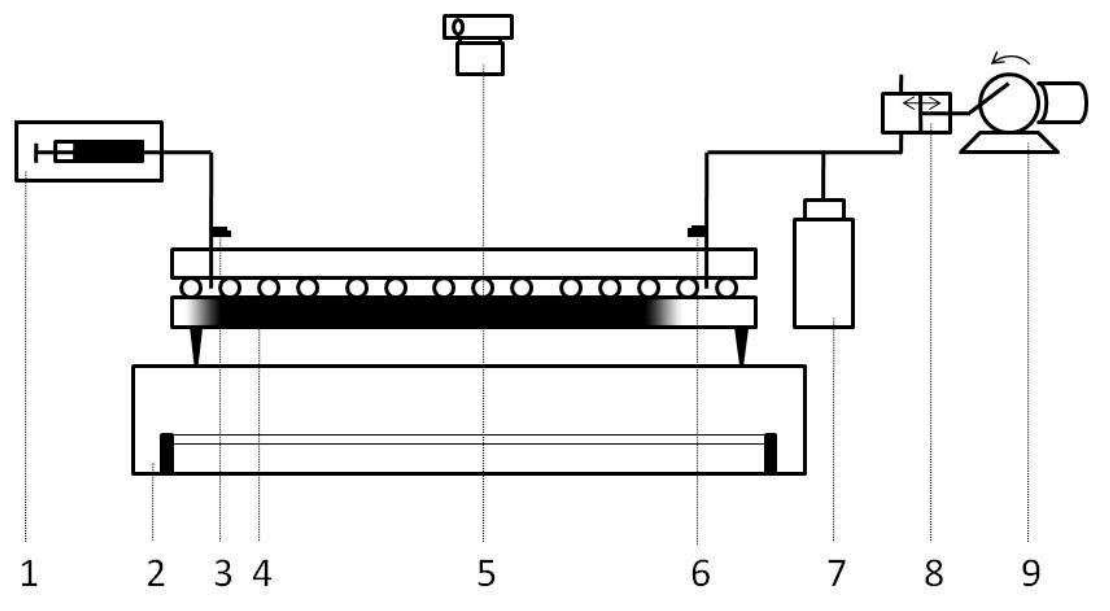

Figure 1. Schematic view of the vertical cross-section of the experimental setup. 1) syringe pump, 2) light box, 3) outlet pressure sensor, 4) porous matrix and the water cushion on the bottom, 5) camera, 6) inlet pressure sensor, 7) container for pressure amplitude regulation, 8) air pump, 9) DC motor that operates the air pump 
keeping the viscosities of the fluids constant throughout experiments (Frette et al. (1997)).

The model construction allows for a certain elasticity. The change in the porous matrix volume and the pressure of the fluid is measured while the matrix is being over-saturated from an external reservoir. The fluid volume in the matrix is

$$
V(p)=V_{0}+\delta V(p)
$$

where $V_{0}=V\left(p_{\text {atm }}\right)$, the volume at the reference pressure (atmospheric pressure), and $\delta V(p)$ is the change in the volume due to the applied over-pressure $\left(\delta p=p-p_{\text {atm }}\right)$. The volume change is found to be linear in the pressure change,

$$
\frac{\delta V(p)}{V_{0}}=\xi \frac{\delta p}{p_{\mathrm{atm}}},
$$

where $\xi$ is the elasticity coefficient of the porous medium. In the pressure range relevant to our experiments its value is measured to $\xi=0.38$. Using Eq. (2) and by expressing the volume as $V(p)=A h(p) \phi(p)$, where $A$ is the area, $h(p)$ the height, and $\phi(p)$ the porosity of the medium, Eq.(1) is rewritten as

$$
\phi(p) h(p)=\phi_{0} h_{0}\left(1+\xi \frac{\delta p}{p_{\text {atm }}}\right),
$$

where $\phi\left(p_{\text {atm }}\right)=\phi_{0}$ and $h\left(p_{\text {atm }}\right)=h_{0}$. This means that the change in the fluid volume due to the imposed pressure results in the alteration of the porosity and the height of the system.

\subsection{BOUNDARY CONDITIONS}

The prime objective with the setup is to study an on-average slow drainage process under oscillatory driving pressure. A custom built pump system is made for this purpose, see Figure 1. Initially, the model is filled with the wetting fluid that is essentially incompressible. During an experiment this fluid is withdrawn from the model at a constant flow rate. The boundary condition on the model outlet is thus defined by the constant outflow rate, corresponding to a constant pressure gradient at the outlet. Without oscillations the inlet side may be left open to air, and during drainage the pressure of the wetting fluid inside the model is lowered with respect to the surrounding atmospheric pressure. In order to oscillate the pressure on the inlet side, the opening is attached to an air cavity of variable size. This cavity is in turn open to surrounding air through a pin-hole, which serves as an air supply and makes the air 


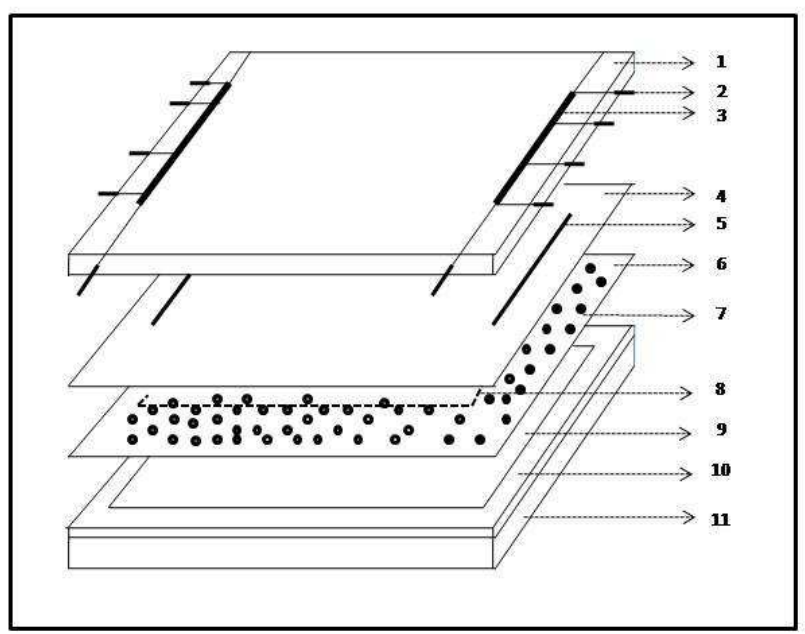

Figure 2. Schematic view of the porous model: 1-upper Plexiglas plate, 2-inlet/outlet tubing connector, 3-inlet/outlet channel, 4-upper adhesive paper sheet, 5- slit, 6-lower adhesive sheet, 7-monolayer of glass beads, 8-silicone filling (defines the geometry of the active matrix), 9-mylar film, 10-aluminum rim and 11-lower Plexiglas plate. Elements 9, 10 and 11 define the pressure cushion.

pressure, on average, equal to the surrounding atmospheric pressure. A larger opening is directly connected to an air pump; a piston that moves back and forth within a cylinder. The motion of the piston compresses and expands the air in the cavity, resulting in an oscillating air pressure at the inlet of the porous matrix. This pressure is monitored, and by tuning the size of the cavity and the piston motion, the desired amplitude of the air pressure oscillations is realized. The oscillatory frequency is given by the piston's frequency.

\subsection{EXPERIMENTAL PROCEDURE}

The porous medium is placed in the horizontal plane over a light box. At the beginning of each experiment the porous matrix is fully saturated with the wetting phase. As the wetting fluid a $80 \%-20 \%$ by mass glycerol-water solution dyed with $0.1 \%$ negrosine is used. The viscosity of the wetting fluid is $\eta_{w}=0.11 \mathrm{~Pa}$ s and the density is $\rho_{w}=1209 \mathrm{~kg} / \mathrm{m}^{3}$. The nonwetting phase is air with viscosity $\eta_{n w}=1.9 \times 10^{-5} \mathrm{Pas}$. The measured value of surface tension between the phases is $\gamma=6.4 \times 10^{-2}$ $\mathrm{N} / \mathrm{m}$. The wetting fluid is withdrawn at a constant rate $Q=0.022 \mathrm{ml} / \mathrm{min}$. The pressure in the nonwetting fluid is oscillated with pressure amplitudes up to $4300 \mathrm{~Pa}$ and frequencies $(f): 0.2 \mathrm{~Hz}, 0.9 \mathrm{~Hz}$, and $4.0 \mathrm{~Hz}$.

The starting point of an experiment is defined as the time when air enters the porous matrix. The invading structure evolves with time 
as the non-wetting fluid advances in the pores occupied by the wetting fluid. The experiment ends when the invading structure reaches the outlet channel. The geometry of the invading cluster at the breakthrough (i.e. when the invading fluid reaches the outlet channel) represents a fingerprint of the drainage process performed under the oscillating pressure conditions at given frequency and amplitude. An example of three different invading structures achieved for three different amplitudes is shown in Figure 3.
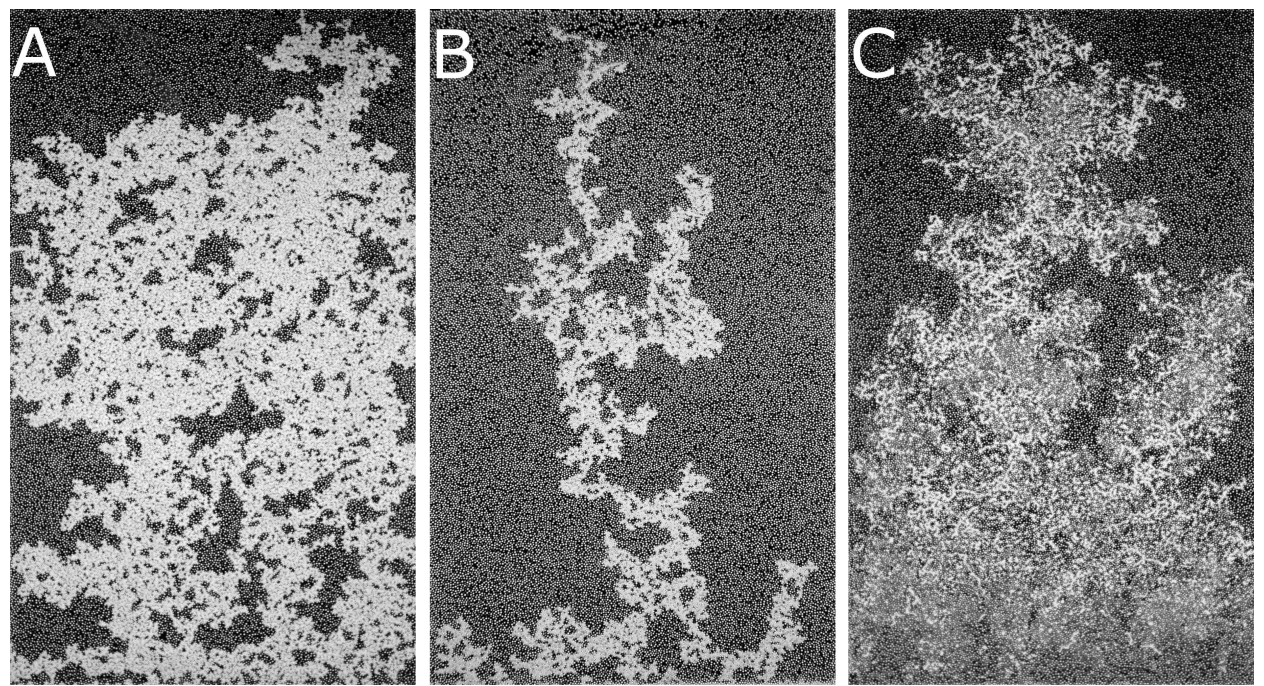

Figure 3. Examples of breakthrough images of the invading cluster created in a drainage process stimulated with the oscillating pressure. The applied frequency is $0.2 \mathrm{~Hz}$ and the amplitudes of the oscillating component vary. The structures shown on the images A, B and $\mathrm{C}$ are created at 20, 560 and $3700 \mathrm{~Pa}$, respectively. The outflow rate of the wetting fluid is $Q=0.022 \mathrm{ml} / \mathrm{min}$. The dark area on the images represents the wetting fluid while the white area is the invading air cluster.

The time evolution of the invasion is recorded by a digital camera (Nikon $D^{70} S$ ) placed above the model. The pictures are taken at three minute time intervals and the resolution of the images is $3008 \times 2000$ pixels (70 pixels/cm).

The pressure is recorded both at the inlet and the outlet. In air a Honeywell 176PCHD214 and in the liquid a Honeywell flow through 26PCA sensor are used. The pressure sensors are connected with a desktop computer through either a Keithley (2000) voltmeter or a National Instruments Data Acquisition (DAQ) card. 


\section{Physics of the process}

In drainage processes the created pattern, the displacement efficiency, and other characteristics of the process depend on the interplay between the forces involved. The relative strength of capillary and viscous forces determines whether a process is capillary fingering or viscous fingering. In non-horizontal systems the role of gravity would have to be taken into account, but it can be neglected for the current study of horizontal flows. In our experiments the oscillatory pressure needs to be compared with the other forces in order to understand its effect. Before turning to this point in Sec. 3.2, we briefly recapitulate some key points about capillary fingering.

On the pore scale, advancement of the displacement front means the invasion of pore after pore, thus overcoming the capillary pressure threshold in each of the invaded pores. The capillary pressure obeys the Young-Laplace law,

$$
p_{c}=p_{n w}-p_{w}=\gamma\left(\frac{1}{R_{1}}+\frac{1}{R_{2}}\right) .
$$

The $p_{n w}$ and $p_{w}$ terms represent the pressures of the nonwetting and the wetting phases, respectively, $\gamma$ is the interfacial tension, and $R_{1}$ and $R_{2}$ are principal radii of curvature. In the experiments the typical pore space is of size $a$, and the capillary pressure is approximated by $p_{c} \approx \gamma / a$.

In a very slow drainage process the front invades the largest pores first since they exert the least resistance. The growth of the displacing cluster is discontinuous (Haines (1930), Morrow (1970), Måløy et al. (1992), Furuberg et al. (1996)) and is characterized by so called bursts or Haines jumps. Before a burst, the pressure builds up until the easiest pore is invaded and subsequently a certain number of pores is occupied at once. The burst lasts as long as the pressure difference in the advancing part of the interface is large enough to overcome the capillary thresholds in the neighboring pores. After the burst the pressure in the invading fluid builds up again until it is large enough to overcome the capillary pressure barrier in another pore throat along the front.

The movement of the fluid through the medium is characterized by the flow velocity, which is related to the viscous pressure gradient by the Darcy law. For the wetting fluid

$$
\mathbf{v}=-\frac{\kappa}{\eta_{w}} \nabla p,
$$

where, $\mathbf{v}$ is so-called seepage or Darcy's velocity and $\kappa$ is the permeability of the system. The ratio between viscous and capillary forces 
is represented through the capillary number. The capillary number is defined as a ratio of the viscous and capillary pressure drop over the pore length,

$$
\mathrm{Ca}=\frac{\Delta p}{p_{c}}=\frac{\eta v a^{2}}{\gamma \kappa} .
$$

Our experiments are performed with a low background speed, so that the capillary number is small enough to keep the flow in the capillary fingering regime (Wilkinson and Willemsen (1983), Lenormand and Zarcone (1985)). However, the capillary number does not include the effects of pressure oscillations. Since the porous medium is elastic, the pressure oscillations cause back and forth motion of the fluids locally, and this alters the local ratio between viscous and capillary forces. An additional dimensionless number is needed to account for this; a number which includes the elastic properties.

\subsection{Pressure Skin-DePth in an elastic, QUASI-TWO-DIMENSIONAL POROUS MEDIUM}

The elasticity of the system allows for some volume changes within each oscillatory cycle. In a much stiffer system the pressure variations would propagate through the entire system, but here the volume changes causes the pressure to attenuate over a certain distance ahead of the front. The distance from the front to which the defending fluid is affected by pressure oscillations is estimated in the following.

For an arbitrary volume of fluid within the porous matrix constrained with $\delta x, \delta y$ and $h(p)$ in $x, y$ and $z$ directions respectively, the mass flow through the vertical surface defined by $\delta y$ and $h(p)$ (assumed perpendicular to the flow) is

$$
\Phi=\rho_{f} \phi(p) h(p) \delta y u .
$$

The $\rho_{f}$ term is the density of the wetting fluid and $u$ is the fluid velocity through the vertical surface. If the designated volume contains fluid of mass $M$, then

$$
M=\rho_{f} \phi(p) h(p) \delta x \delta y,
$$

which means that the surface density $\rho_{s}=M /(\delta x \delta y)$ becomes

$$
\rho_{s}=\rho_{f} \phi(p) h(p) .
$$

The mass conservation law for the surface density is

$$
\partial_{t}\left(\rho_{s}\right)+\nabla \cdot\left(\rho_{s} \mathbf{u}\right)=0 .
$$

Note that the actual fluid velocity $\mathbf{u}$, which is related to the Darcy velocity $\mathbf{v}$ as v $/ \phi(p)$ (Anghel et al. (2006), Flekkøy et al. (2001)), enters 
here and yields

$$
\partial_{t}\left(\rho_{f} \phi(p) h(p)\right)+\nabla \cdot\left(\rho_{f} \phi(p) h(p) \frac{\mathbf{v}}{\phi(p)}\right)=0 .
$$

By inserting the expressions for $\phi(p) h(p)$ and $\mathbf{v}$, Eq. (3) and Eq. (5), respectively, we obtain

$$
\partial_{t}\left(\rho_{f} \phi_{0} h_{0}\left[1+\frac{\xi \delta p}{p_{\mathrm{atm}}}\right]\right)-\nabla \cdot\left(\rho_{f} h(p) \frac{\kappa}{\eta_{w}} \nabla p\right)=0 .
$$

Due to the incompressibility of the fluid $\rho_{f}$ is considered constant. In the time derivative term the pressure $p$ in $\delta p=p-p_{\text {atm }}$ is the only time dependent quantity. In the spatial derivative $\kappa, h(p)$, and $\nabla p$ are all in principle position dependent. However, we approximate this by assuming the permeability $\kappa$ to be constant and claiming that the spatial variation in height $\left(\xi h_{0} \delta p / p_{\text {atm }}=h(p)-h_{0}\right)$ in Eq. (12) is negligibly small compared to the model thickness $h_{0}$; therefore $h(p) \approx$ $h_{0}$. This gives

$$
\frac{\rho_{f} \phi_{0} h_{0} \xi}{p_{\text {atm }}} \partial_{t} p-\frac{\rho_{f} \kappa h_{0}}{\eta_{w}} \nabla \cdot \nabla p=0 .
$$

Rearranging the terms, Eq. (13) can be written in the form of a diffusion equation

$$
\partial_{t} p=D \nabla^{2} p
$$

with the diffusion constant

$$
D=\frac{\kappa p_{\text {atm }}}{\eta_{w} \phi_{0} \xi}
$$

In order to estimate the pressure skin-depth in the direction of flow, we solve Eq. (14) assuming invariance in the transverse direction; i.e. $p=p(x, t)$. The boundary conditions are i) for the inlet: $p(0)=$ $P \cos (\omega t)+p_{\text {atm }}$, where $P$ and $\omega=2 \pi f$ are the amplitude and angular frequency of the applied oscillation and ii) for the outlet - taking the limit at infinity: $\nabla p_{\infty}=-v_{0} \eta_{w} / \kappa$.

The solution for $p(x, t)$ (which may be verified by direct inspection) is the real part of

$$
p=P e^{i(k x-\omega t)}+p_{\text {atm }},
$$

where the wave number is

$$
k=\frac{\sqrt{2}}{2}(1+i) \sqrt{\frac{\omega \eta_{w} \phi_{0} \xi}{p_{\mathrm{atm}} \kappa}} .
$$


The imaginary part of $k$ gives the damping. The length over which the pressure is reduced to $1 / e$ is thus

$$
x_{s}=\frac{1}{\operatorname{Im} k}=\sqrt{\frac{2 p_{\text {atm }} \kappa}{\omega \eta_{w} \phi_{0} \xi}} .
$$

This pressure skin-depth depends on the frequency of the oscillations. The behavior of the fluid pressure ahead of the invasion front is illustrated in Figure 4.

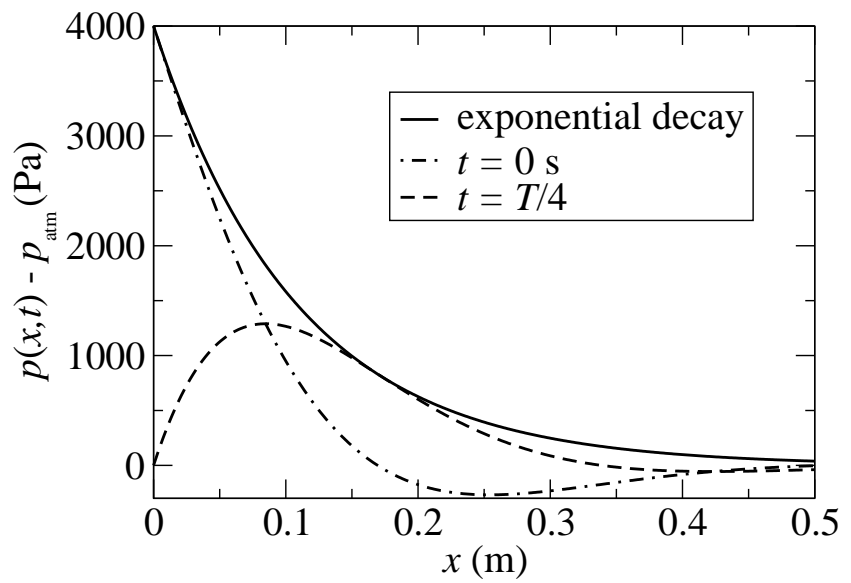

Figure 4. Pressure wave propagation in the defending fluid as a function of the distance ahead of the invasion front. The dash-dot line represents the decay of the pressure amplitude with distance at time $t=0$, the dashed line represents the pressure decay with distance at $t=T / 4(T=1 / f)$ and the solid line represents the exponential decay of the pressure oscillation amplitude(envelope). The initial amplitude of the oscillation is $4000 \mathrm{~Pa}$ and the frequency is $0.2 \mathrm{~Hz}$.

\subsection{Fragmentation NUMBer}

Due to the pressure oscillations and elasticity there is back and forth movement of the front (Figure 5). The forward-backward displacement of the front within each pressure oscillatory cycle will result in fragmentation of the invading cluster if the displacement is large enough.

The fragmentation generally occurs when the menisci move backwards within an oscillatory cycle. The likeliness of the fragmentation is higher when the frontal displacement is large relative to the typical pore size, hence the fragmentation number is defined as a ratio of the backward traveling distance $\left(\Delta x^{-}\right)$and the pore size $(a)$

$$
F=\frac{\Delta x^{-}}{a} .
$$




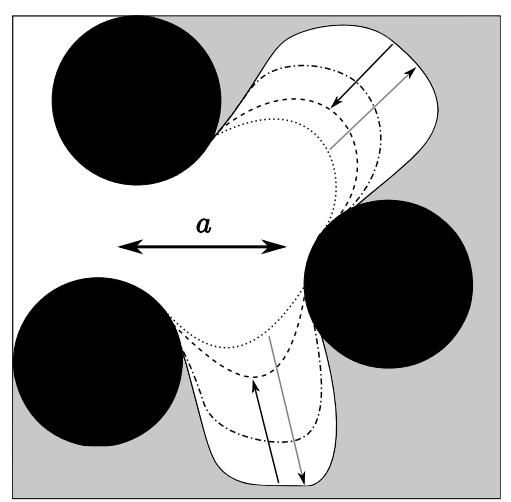

Figure 5. Schematic view of frontal displacement due to the imposed pressure oscillations on the pore scale. The gray arrow represents the direction of the forward displacement $\left(\Delta x^{+}\right)$. The black arrow represents the direction of the backward displacement of the front $\left(\Delta x^{-}\right)$. The dashed lines schematically show successive positions of the front as it is being displaced due to the pressure oscillations in the nonwetting phase.

The backward traveling distance can be expressed as

$$
\Delta x^{-} \approx \frac{1}{\omega} \frac{\kappa P}{\eta_{w} x_{s}},
$$

where the first term represents the time scale of the motion and the second term is an approximation of the velocity of the meniscus. The fragmentation number

$$
F=\frac{1}{\omega} \frac{\kappa P}{\eta_{w} x_{s} a}=\sqrt{\frac{P^{2} \kappa \phi_{0} \xi}{4 \pi f \eta_{w} p_{\text {atm }} a^{2}}}=c \frac{P}{\sqrt{f}} .
$$

is then proportional to the pressure oscillation amplitude and inversely proportional to the square root of the frequency. In our experiments the model and fluid parameters (i.e. elasticity coefficient, permeability, pore size, porosity, viscosity) are held constant, giving $c=5.7 \cdot 10^{-5}$ $\left(\mathrm{Pa}^{-1} \mathrm{~s}^{-1 / 2}\right)$ in Eq.(21).

To relate our fragmentation number to a real world situation we resort to Biot's (Biot (1962)) theory of pressure wave propagation in a fluid-saturated porous material. As shown in Pride (2005), similarly to our case, pressure propagation in a porous material is described by a diffusion equation with the diffusion coefficient $D_{r}$. For the situations where a considerable contribution to the compressibility comes from the soft fraction in the porous medium (e.g. fluid with possibly trapped air bubbles), the diffusion coefficient can be written as

$$
D_{r}=\frac{\kappa_{r}}{\beta \eta}
$$


where, $\kappa_{r}$ is the permeability of the porous medium, $\beta$ is the compressibility and $\eta$ is the viscosity of the soft medium. Analogously to our quasi-two dimensional case the fragmentation number for a real, three-dimensional porous material can be calculated as:

$$
F=\frac{\kappa_{r} P}{a \eta \sqrt{2 D_{r} \omega}} .
$$

Consider a seismic wave with a pressure amplitude $P=10^{3} \mathrm{~Pa}$ and frequency of $1 \mathrm{~Hz}$, corresponding to a moderate seismic event or to an oscillation created artificially in a nearby well (Shearer (1999)). For a porous material filled with water where the compressibility of water is $\beta=10^{-10} \mathrm{~Pa}^{-1}$, and the permeability of the solid matrix is $\kappa_{r}=10^{-11}$ $\mathrm{m}^{2}$, with pores of size $a=10^{-5} \mathrm{~m}$, using Eq. 23 the calculated value of $F$ is 0.07 . This value is in the same range as the values calculated for the synthetic porous medium used in the experiments.

\section{Results and discussions}

The design of the experimental set-up allows independent tuning of amplitudes and frequencies of the pressure oscillations. The oscillatory frequency and the amplitude are chosen at the beginning and kept fixed throughout each experiment. In this study three different frequencies and a relatively large range of amplitudes are used. The frequencies are $0.2,0.9$ and $4 \mathrm{~Hz}$. For each frequency the amplitudes range from around 20 to approximately $4000 \mathrm{~Pa}$. The typical capillary pressure for the set-up used in the study is around $60 \mathrm{~Pa}$. For each used frequency there is also a characteristic pressure skin-depth. The skin-depths are $10.7 \mathrm{~cm}, 5.0 \mathrm{~cm}$ and $2.4 \mathrm{~cm}$ for $0.2,0.9$ and $4 \mathrm{~Hz}$, respectively (see Eq. (18)).

The outflow rate is kept low thus minimizing the effects of the viscous forces and maintaining the background flow of the fluid in the capillary regime. Lower values of $Q$ would not affect the morphology of the invading cluster. On the contrary, a considerable increase in $\mathrm{Q}$ would lead to a crossover of the background flow from the capillary to the viscous regime therefore affecting the morphology before the stimulation is even applied (Løvoll et al. (2004)).

The objective of the study is the analysis of the effects of the pressure oscillations on the invading cluster geometry at break-through, when the invading cluster reaches its maximal size. The breakthrough images are shown in Figure 6.

In general, it is observed that the applied oscillations change the overall geometry of the invading cluster. The extent of the change 


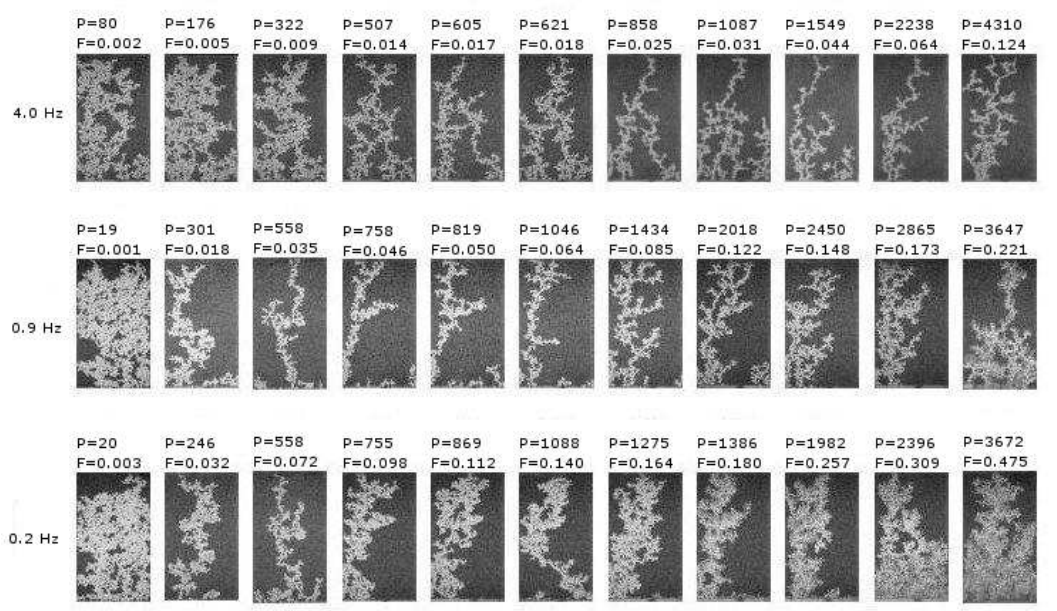

Figure 6. Breakthrough images of all experiments. The numbers above individual images indicate the amplitude of the pressure oscillations given in $\mathrm{Pa}$ (top row) and the fragmentation number $(F)$ in the bottom row. The numbers $0.2,0.9$ and 4.0 refer to the frequency of the oscillations $(\mathrm{Hz})$ at which the experiments are carried out. The flow direction is from the bottom of each individual image and up.

relative to the geometry of the invading cluster in a non-stimulated drainage strongly depends on the chosen combination of the amplitudes and the frequencies of the oscillation (see Figure 3). There are four different flow regimes identified. Each of the regimes is characterized by a typical morphology of the invading cluster. To classify the geometrical characteristics of each regime the global saturation $\left(S_{n w}\right)$ with the non-wetting fluid at breakthrough is measured. To characterize the stimulation, the fragmentation number $F$ is also evaluated in each case.

\subsection{SATURATION}

The differences in the geometry of the invading cluster for different flow regimes largely reflect in the non-wetting saturation $\left(S_{n w}\right)$ at breakthrough. The outflow rate $(Q)$ and the fragmentation number $(F)$ can be tuned independently. In all experiments the outflow rate is kept constant. For this constant outflow rate the saturation depends on the fragmentation number and also on the breakthrough time $\left(t_{b}\right)$ since $S_{n w}=Q t_{b} / V_{0} \phi$. The plots of the saturation as a function of the fragmentation number $\left(S_{n w}(F)\right)$ are given in Figure 7.

The data collapse suggests that the fragmentation number is the controlling parameter. Different levels of saturation in the porous medium can be achieved by choosing the amplitude and frequency of the os- 


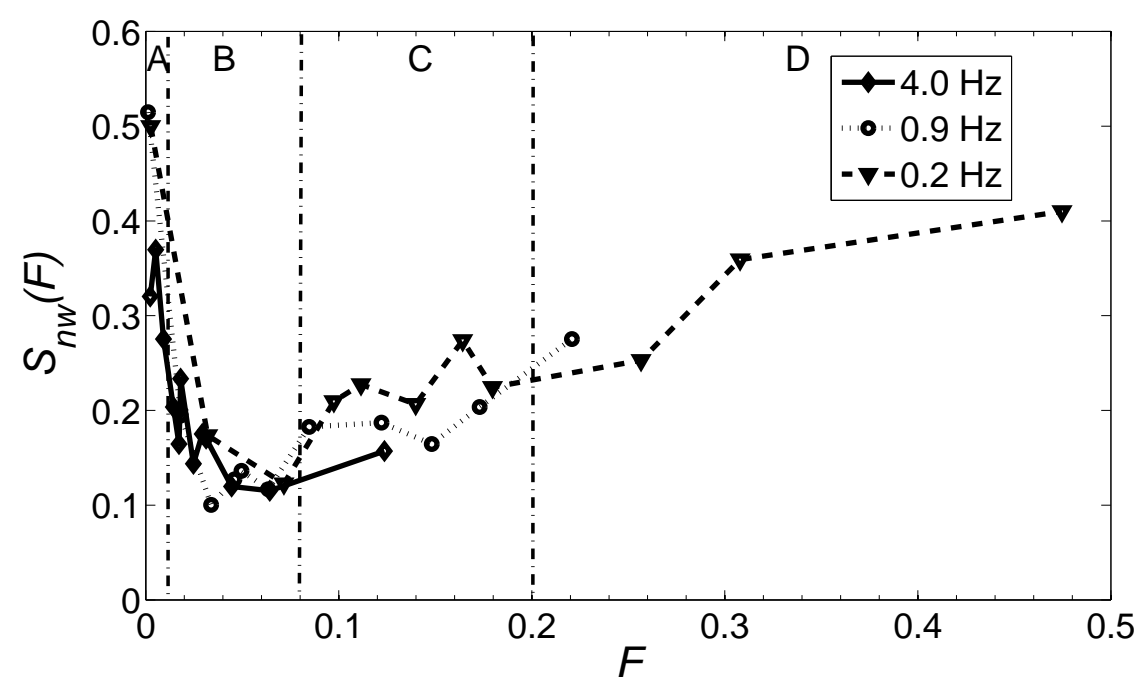

Figure 7. Saturation (ratio of the matrix volume occupied with the non-wetting fluid to the total volume of the voids) as a function of the fragmentation number $\left(S_{n w}(F)\right)$. The solid line connects data points from the experiments where the oscillatory frequency is $4.0 \mathrm{~Hz}$, the dotted line marks the $0.9 \mathrm{~Hz}$ experiments and the dashed line connects the data points from the $0.2 \mathrm{~Hz}$ experiments. The vertical (dash-dot) lines divide the graph into sections that correspond to different flow regimes (A-capillary fingering, B-fingering, C-fragmentation, D-foam formation).

cillations to match the fragmentation number that corresponds to the desired saturation.

\subsection{Phase Diagram and invading Cluster morphology}

The classification of the flow regimes is based on the fragmentation number and is summarized on a phase diagram given in Figure 8.

\subsubsection{A: Capillary fingering}

In the experiments performed in a relatively low amplitude regime the breakthrough clusters resemble the structures typically observed in the so-called capillary fingering regime (Figure 9), in which the pore throats with the lowest capillary pressure thresholds are invaded first. This behavior is somewhat expected because the backwards traveling distance of the menisci is much smaller than the average pore size. In these cases the impact of the pseudo-seismic stimulation is low or non-existing. This sort of geometry typically occurs in the experiments where $F$ is up to 0.01, independently of the applied frequency. 


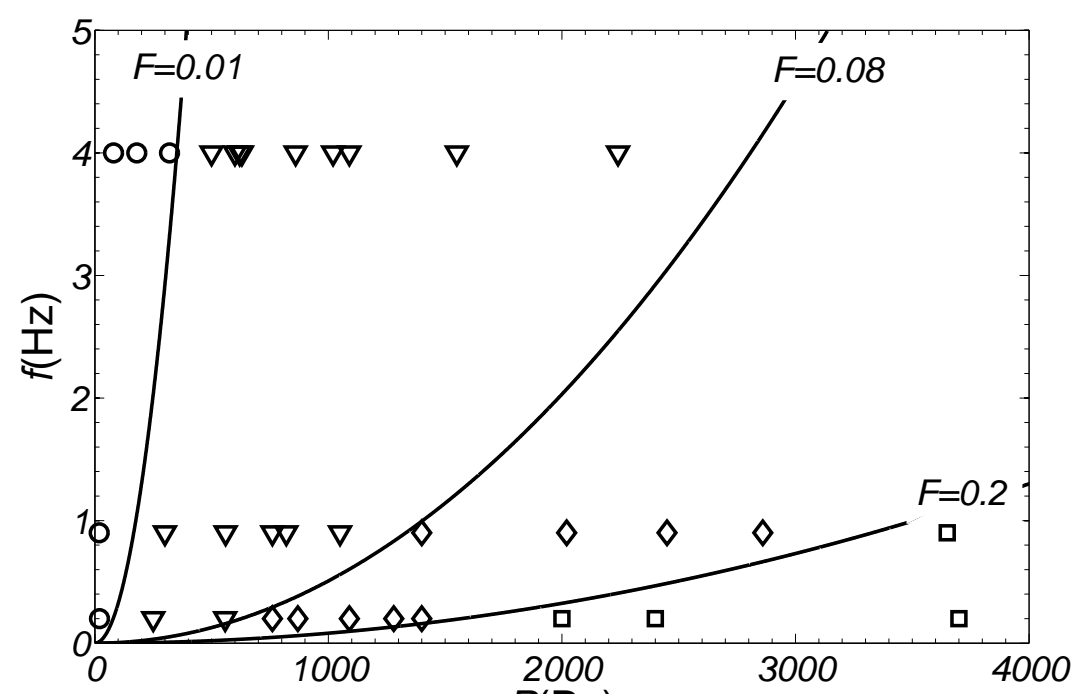

Figure 8. Phase diagram. The solid lines represent the borders between the phases based on the calculated values of the fragmentation number $\left(f=\left(\frac{c P}{F}\right)^{2}\right)$. Different symbols represent four geometries of the invading cluster (0: capillary fingering, $\nabla$ : fingerlike structure, $\diamond$ : fragmented structure and $\square$ : structure where foam is present).

\subsubsection{B: Fingerlike geometry}

The fingerlike structures were typically achieved for $F^{\prime} s$ ranging from 0.01 to 0.08 . Generally the structure is either a result of a simultaneous growth of multiple fingers on different parts of the invading front or just a single finger that spans the matrix. The single fingerlike geometries resemble viscous fingering (Figure 10). Regardless of their number, there is also a noticeable difference in the thickness of the individual fingers when the amplitude is changed. The thickest fingers are obtained for relatively low amplitude experiments. As an example, images from two experiments performed at different fragmentation numbers are shown in Figure 11.

A similar increase in thickness is observed when the speed of a drainage process in the viscous regime without oscillations is decreased (Toussaint et al. (2005)).

\subsubsection{C: Fragmented geometry}

Another prominent feature is the fragmentation or discontinuation of the invading cluster. The fragmentation is present in the cases when $F$ is larger than 0.08. Typically the fragmentation takes place along the front line and is particularly pronounced ahead of the most advanced part of the front. Initially, the fragmented structures largely resemble the single-finger like structures with branches that laterally grow off the 


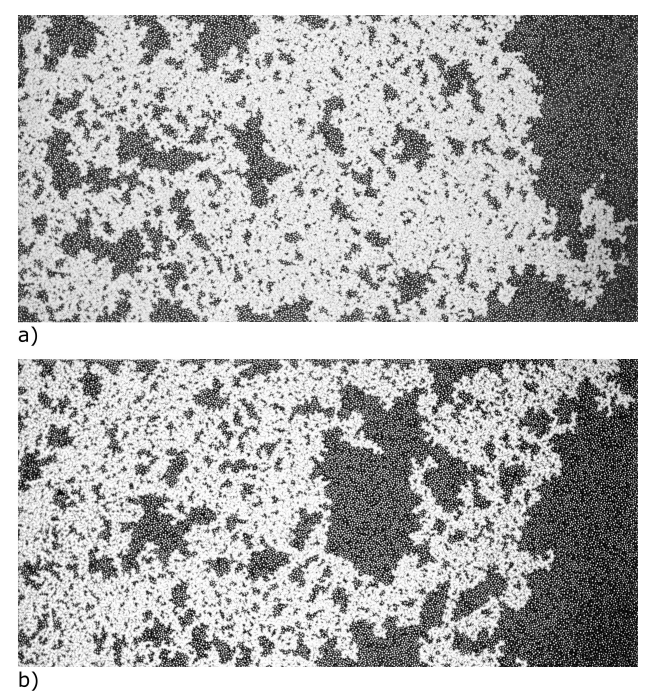

Figure 9. Comparison of an invading cluster geometry achieved in a low amplitude and frequency experiment (a) with typical capillary fingering structure (b). The stimulation tends to generate more compact invading structures (smaller sizes of the trapped clusters) than those characteristic for the capillary fingering regime. The direction of the flow is from the left- to the right-hand side.

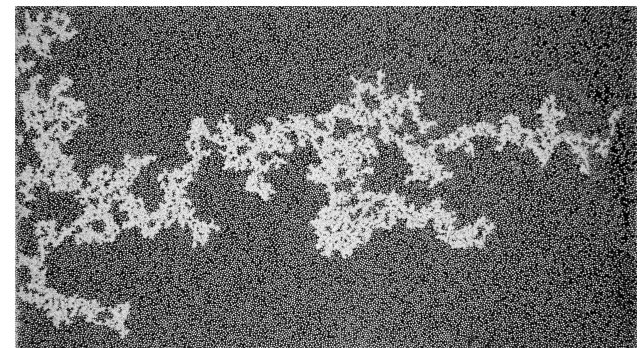

Figure 10. Example of a single-finger like invading cluster. The displacing structure is elongated and typically have relatively low number of branches that grow sideways with respect to the main finger. The thickness of the fingers varies and is generally larger than the thickness of the fingers observed in the viscous regime. The direction of the flow is from the left- to the right-hand side.

main structure. The level of fragmentation depends on $F$. For the $F$ values on the higher end of the spectrum (values closer to 0.2 ) the effects of fragmentation are very well visible. A comparison of two fragmented invading structures with the different $F$ values is shown in Figure 12.

\subsubsection{D: Geometries with foam}

Finally, when $F$ exceeds 0.2 fragmentation dominates the invasion and small air bubbles are introduced in the wetting phase. The trapping of the air bubbles in the defending fluid is defined as foam formation. In 


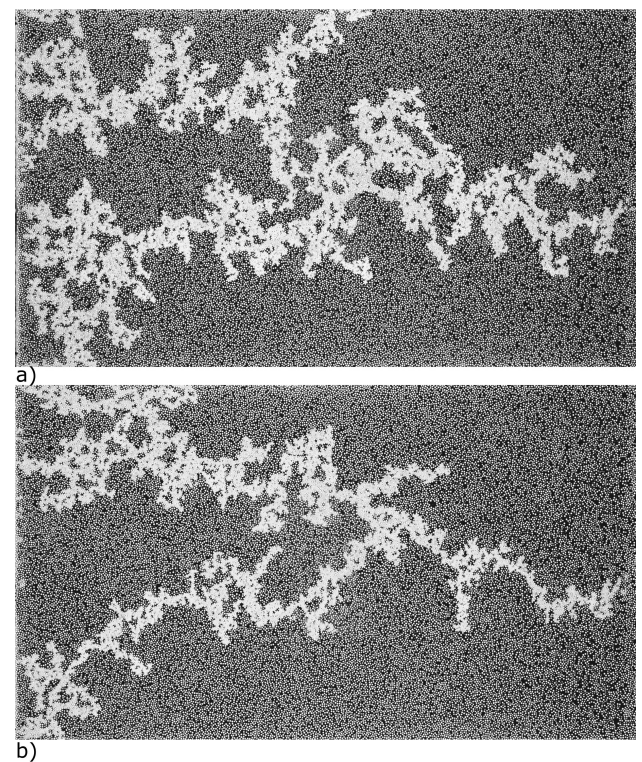

Figure 11. Comparison of two finger-like structures achieved at $F=0.018$ (a) and $F=0.025$ (b) in the $4 \mathrm{~Hz}$ series. The thickness of the fingers is distinguishable while the general shape is preserved to a great extent. The direction of the flow is from the left- to the right-hand side.

the current set-up and the range of achievable fragmentation numbers the foam formation is present in a very few cases and related to $F^{\prime} s$ larger than 0.2 , which corresponds to the largest amplitude experiments in the 0.2 and $0.9 \mathrm{~Hz}$ series. Typically foam forms along the front line but is being left behind as the invading cluster grows and fragments. In the cases where a considerable amount of foam is formed there is a lack of a well connected invading cluster and a clearly distinguishable front line due to excessive fragmentation. The displacing structure appears as a cloud-like formation. A typical example of a cluster with a considerable amount of foam is shown in Figure 13.

\section{Conclusion}

The effects of pseudo-seismic stimulation on a slow quasi two-dimensional drainage process in an elastic porous medium are addressed. It is found that the pressure stimulation of the drainage process alters the flow regime in which the wetting fluid is evacuated from the porous medium. The change in the flow regime due to applied pressure oscillation results in a change of the morphological characteristics of the invading structure. The degree of the impact greatly depends on the intensity of the 


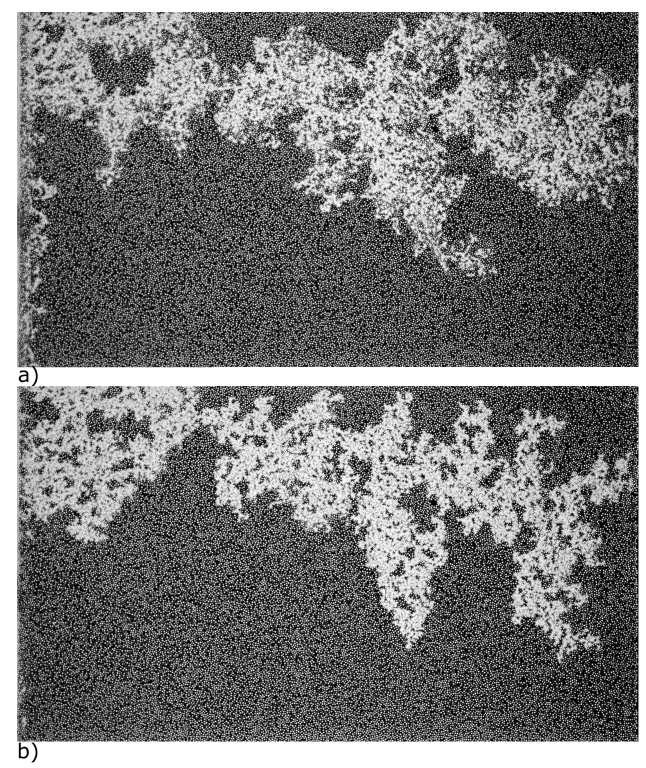

Figure 12. Difference in the level of fragmentation depending on the $F$ values. The example $(a)$, represents a displacement cluster achieved for $F=0.18$. The entire structure is heavily disconnected and the fragmentation is not localized but rather present in the entire displacing structure. In the example $(b)$, the value of $F$ is on its lower end $(F=0.098)$. The level of fragmentation is very low and the fragments are located mostly around the top part of the invading cluster. The direction of the flow is from the left- to the right-hand side.

stimulation and the elasticity of the porous medium. The stimulation consisting of the oscillatory amplitudes and frequencies that result in small incursions of the oscillating menisci into the wetting phase has a very little or no impact on the geometry of the displacing structure. On the contrary, the stimulation that produces larger incursion of the menisci within an oscillatory cycle leads to a dramatic change in the flow regime. The difference in the flow regimes then results in a notably different geometries of the invading structure.

There are four distinguishable flow regimes followed with four regime specific geometries for the used range of amplitudes and frequencies. The classification of the observed morphologies is done by using the proposed fragmentation number and the characteristic level of residual saturation (at breakthrough). The phase diagram shows that the proposed fragmentation number can be used to differentiate and characterize the flow regimes. The morphological differences of the invading structures for different regimes result in various levels of saturation with non-wetting fluid. The data collapse obtained when the residual 


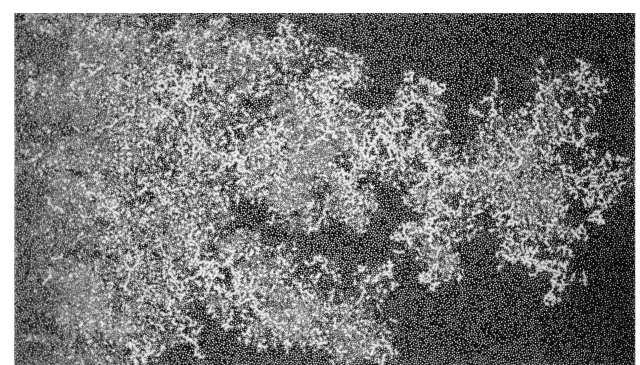

Figure 13. Example of a displacement cluster with foam. This experiment is carried out at the maximal $F$ achievable for the described experimental set-up. The amount of formed foam varies from experiment to experiment and depends on the fragmentation number. In the cases where the $F$ values were relatively smaller the foam forms in a smaller quantity. The direction of the flow is from the left- to the right-hand side

saturation is plotted as function of the fragmentation number suggests that the fragmentation number is the relevant controlling parameter.

\section{Acknowledgment}

M.Jankov, G. Løvoll and H. A. Knudsen acknowledge support from NFR (Norwegian Research Council) through a PETROMAKS grant. R. Planet acknowledges Ministerio de Ciencia y Tecnologa (Spain) for a grant associated to the project BQU2003-05042-C02-02.

\section{References}

Aker, E., K. J. Måløy, and A. Hansen: 2000, 'Viscous stabilization of 2D drainage displacements with trapping'. Physical Review Letters 84, 4589-4592.

Anghel, D.-V., M. Strauss, E. G. Flekkøy, S. McNamara, and K. J. Måløy: 2006, 'Structure formation and instability in a tube of sand (vol 87, pg 134302, 2001)'. Physical Review Letters 97, 059902.

Beresnev, I. A. and P. A. Johnson: 1994, 'Elastic-wave stimulation of oil production: A review of methods and results'. Geophysics 59, 1000-1017.

Beresnev, I. A., R. D. Vigil, W. Q. Li, W. D. Pennington, R. M. Turpening, P. P. Iassonov, and R. P. Ewing: 2005, 'Elastic waves push organic fluids from reservoir rock'. Geophys. Res. Lett. 32, L13303.

Biot, M. A.: 1962, 'Mechanics of Deformation and Acoustic Propagation in Porous Media'. Journal of Applied Physics 33(4), 1482-1498.

Birovljev, A., L. Furuberg, J. Feder, T. Jøssang, K. J. Måløy, and A. Aharony: 1991, 'Gravity invasion percolation in 2 dimensions - experiment and simulation'. Physical Review Letters 67, 584-587.

Chandler, R., J. Koplik, K. Lerman, and J. F. Willemsen: 1982, 'Capillary displacement and percolation in porous media'. Journal of Fluid Mechanics 119, 249-267. 
Dullien, F. A. L.: 1992, Porous Media Fluid Transport and Pore Structure. Academic Press, San Diego.

Flekkøy, E. G., S. McNamara, K. J. Måløy, and D. Gendron: 2001, 'Structure Formation and Instability in a Tube of Sand'. Physical Review Letters 87, 134302.

Fountain, J. C., A. Klimek, M. G, Beikirch, and T. M. Middleton: 1991, 'The use of surfactants for in situ extraction of organic pollutants from a contaminated aquifer'. Journal of Hazardous Materials 28, 295-311.

Frette, O. I., K. J. Måløy, and J. Schmittbuhl: 1997, 'Immiscible displacement of viscosity-matched fluids in two-dimensional porous media'. Physical Review E 55, 2969-2975.

Furuberg, L., K. J. Måløy, and J. Feder: 1996, 'Intermittent behavior in slow drainage'. Physical Review E 53, 966-977.

Haines, W. B.: 1930, 'Studies in the physical properties of soil. V. The hysteresis effect in capillary properties, and the modes of moisture distribution associated therewith'. J. Agr. Sci. 20, 97-116.

Hilpert, M.: 2007, 'Capillarity-induced resonance of blobs in porous media: Analytical solutions, Lattice-Boltzmann modeling, and blob mobilization'. Journal of Colloid and Interface Science 309, 493-504.

Hilpert, M., G. H. Jirka, and E. J. Plate: 2000, 'Capillarity-induced resonance of oil blobs in capillary tubes and porous media'. Geophysics $\mathbf{6 5}, 874-883$.

Iassonov, P. P. and I. A. Beresnev: 2003, 'A model for enhanced fluid percolation in porous media by application of low-frequency elastic waves'. J. Geophys. Res. 108, 2138

Lenormand, R., E. Touboul, and C. Zarcone: 1988, 'Numerical models and experiments on immiscible displacement in porous media'. Journal of Fluid Mechanics 189, 165-187.

Lenormand, R. and C. Zarcone: 1985, 'Invasion Percolation in an Etched Network: measurement of a Fractal Dimension'. Physical Review Letters 54, 2226-2229.

Lenormand, R. and C. Zarcone: 1989, 'Capillary Fingering: Percolation and Fractal Dimension'. Transport in Porous Media 4, 599-612.

Lenormand, R., C. Zarcone, and A. Sarr: 1983, 'Mechanisms of the displacement of one fluid by another in a network of capillary ducts'. Journal of Fluid Mechanics 135, 337-353.

Li, W. Q., R. D. Vigil, I. A. Beresnev, P. Iassonov, and R. Ewing: 2005, 'Vibrationinduced mobilization of trapped oil ganglia in porous media: Experimental validation of a capillary-physics mechanism'. Journal of Colloid and Interface Science 289, 193-199.

Løvoll, G., Y. Méheust, K. J. Måløy, E. Aker, and J. Schmittbuhl: 2005, 'Competition of gravity, capillary and viscous forces during drainage in a two-dimensional porous medium, a pore scale study'. Energy 30, 861-872.

Løvoll, G., Y. Méheust, R. Toussaint, J. Schmittbuhl, and K. J. Måløy: 2004, 'Growth activity during fingering in a porous Hele-Shaw cell'. Physical Review E 70, 026301.

Måløy, K. J., J. Feder, and T. Jøssang: 1985, 'Viscous Fingering Fractals in Porous Media'. Physical Review Letters 55, 2688-2691.

Måløy, K. J., L. Furuberg, J. Feder, and T. Jøssang: 1992, 'Dynamics of slow drainage in porous-media'. Physical Review Letters 68, 2161-2164.

Méheust, Y., G. Løvoll, K. J. Måløy, and J. Schmittbuhl: 2002, 'Interface scaling in a two-dimensional porous medium under combined viscous, gravity and capillary effects'. Physical Review E 66, 051603. 
Morrow, N. R.: 1970, 'Physics and Thermodynamics of Capillary Action in Porous Media'. Industrial and Engineering Chemistry 62, 32-56.

Pennell, K. D., M. Q. Jin, L. M. Abriola, and G. A. Pope: 1994, 'Surfactant enhanced remediation of soil columns contaminated by residual tetrachloroethylene'. Journal of Contaminant Hydrology 16, 35-53.

Pride, S. R.: 2005, Relationship between seismic and hydrological properties in Hydrogeophysics edited by Rubin Y. and Hubard S. S. Springer Netherlands.

Pride, S. R., E. G. Flekkøy, and O. Aursjø: 2008, 'Seismic stimulation for enhanced oil recovery'. Geophysics 73, 023-035.

Reddi, L. N. and S. Challa: 1994, 'Vibratory Mobilization of Immiscible Liquid Ganglia in Sands'. Journal of Environmental Engineering 120, 1170-1190.

Roberts, P. M., I. B. Esipov, and E. L. Majer: 2003, 'Elastic-wave stimulation of oil reservoirs: Promising EOR technology?'. The Leading Edge 22, 448-453.

Roberts, P. M., A. Sharma, V. Uddameri, M. Monagle, D. E. Dale, and L. K. Steck: 2001, 'Enhanced DNAPL transport in a sand core during dynamic stress stimulation'. Environmental Engineering Science 18, 67-79.

Saffman, P. G. and G. Taylor: 1958, 'The penetration of a fluid into a porous medium or helle-shaw cell containing a more viscous fluid'. Proc. Roy. Soc. A245, 312329.

Shearer, P. M.: 1999, Introduction to seismology. Cambridge University Press.

Toussaint, R., G. Løvoll, Y. Méheust, K. J. Måløy, and J. Schmittbuhl: 2005, 'Influence of pore-scale disorder on viscous fingering during drainage'. Europhysics Letters 71, 583-589.

Wagner, G., A. Birovljev, P. Meakin, J. Feder, and T. Jøssang: 1997, 'Fragmentation and migration of invasion percolation clusters: Experiments and simulations'. Physical Review E 55, 7015-7029.

Wilkinson, D.: 1984, 'Percolation model of immiscible displacement in the presence of buoyancy forces'. Physical Review A 30, 520-531.

Wilkinson, D. and J. F. Willemsen: 1983, 'Invasion percolation: a new form of percolation theory'. Journal of Physics A: Mathematical and General 16, $3365-3376$. 